\title{
Studying the Nearby Universe with Chandra
}

\author{
Q. Daniel Wang \\ Astronomy Department, University of Massachusetts, Amherst, MA \\ 01003, USA
}

\begin{abstract}
I highlight results from Chandra observations of nearby galaxies, including the Milky Way. These observations have offered insights into old mysteries and indications of new high energy astrophysical phenomena and processes that are yet to be understood.
\end{abstract}

\section{Introduction}

X-ray emission is an excellent tracer for high temperature and/or high velocity activity in galaxies. Such high energy activity is largely produced by the endproducts of massive stars and by supermassive black holes in galactic centers (e.g., Helfand 1984). Neutron stars, stellar mass black holes, supernova remnants (SNRs), and the diffuse hot interstellar medium (ISM), as well as active nuclei (AGNs) are often best studied in X-ray. It is not unusual that such objects or gaseous features leave no trace at all in other wavelength bands. The study of massive star end-products, in particular, is important to the understanding of the interplay between stars and the ISM, or the "ecosystem" of galaxies. This is also intimately tied to the thermal and chemical feedback from galaxies to the intergalactic medium (IGM). Significant X-ray emission may also arise from many other processes such as magnetic field reconnection and the bremsstrahlung of low energy cosmic rays.

Chandra is a unique X-ray observatory that for the first time provides us with the capability to obtain X-ray images with arcsecond resolution, broad energy coverage $(0.2-10 \mathrm{keV})$, and good CCD spectral resolution $(\sim 130 \mathrm{eV})$. The data obtained with Chandra is revolutionizing our view of the $\mathrm{X}$-ray universe. In this writing-up of my overview talk, I will concentrate on various new high energy astrophysical phenomena revealed by Chandra ACIS observations, particularly on topics that are not covered (adequately) by other speakers. I will start with our Galactic center region and then move on to nearby galaxies, making connections to the evolving universe that Professor Hasinger will be reviewing next.

\section{The Milky Way Center as a Laboratory for High Energy Astro- physics}

Nuclear regions of galaxies are the mecca of high energy phenomena and processes, which are manifested by AGNs and starbursts. The best site for a detailed study of the complex interaction of such energetic activity and its interaction 
with the unique nuclear environment is our own Galactic center (GC) region at a distance of only $8 \mathrm{kpc}$.

An overall picture of the GC region as a mildly active starburst galaxy has emerged. Three young massive stellar clusters have been discovered (Arches, Galactic center, and Quintuplet; e.g., Figer et al. 1999). Massive stars $(M \gtrsim$ $\left.10 M_{\odot}\right)$ themselves are energetic objects, releasing fast stellar winds and large amounts of ionizing photons. Such stars are also short-lived and explode as supernovae on time-scales from a few to a few tens of million years, depending of their masses. Stellar remnants of the explosions (neutron stars and black holes) can often be seen as pulsars and X-ray binaries, whereas supernova blastwaves can naturally heat ambient gas to temperatures of million degrees or higher. Radio and infrared observations have already revealed luminous shells or arcs as well as nonthermal filaments (NTFs) with magnetic field strengths possibly as high as $\sim 1 \mathrm{mG}$ (e.g., Morris 1994). These features, uniquely seen in the GC region, demonstrate the strong interaction between massive stars and the extreme high density and high magnetic field environment. Chandra has provided us with a new tool to probe the high-energy properties of the GC.

Wang, Gotthelf, \& Lang (2002) have carried out a systematic survey of a $2 \times 0.8$ square degree field, which consists of 30 overlapping Chandra ACIS-I observations (about $11-12 \mathrm{ks}$ exposure each) and covers the most active ridge around the GC (Fig. 1). The survey, complemented by deeper pointings $(z 50$ ks each) on several specific targets with the same instrument (Yusef-Zadeh et al. 2002; Takagi, Murakami, \& Koyama 2002; Baganoff et al. 2001), provides us with the first high resolution X-ray view of the GC with sensitivity more than two orders of magnitude higher than any pre-Chandra observations (e.g., $A S C A)$. While the Chandra data analysis is still ongoing, I summarize some of the preliminary results.

\subsection{A Global X-ray View}

Fig. 1 includes a broad-band X-ray contour image constructed with the data from the GC ridge survey. Close to $10^{3}$ discrete sources are detected. Only a handful of these X-ray sources are previously known. The brightest source 1E 1740.729.42 is a "micro quasar", which contains an accreting stellar mass black hole and extended radio jets. The second brightest source 1E 1743.1-2842 is a lowmass X-ray binary, possibly with a neutron star as the primary. SNR G0.9+0.1 is a composite supernova remnant with an X-ray-emitting Crab-like nebula and a larger-scale radio shell. Several of the other luminous X-ray sources detected previously were relatively faint during the Chandra survey. GRO J1744-28 and SAX J1747.0-2853 are two examples (Wijnands \& Wang 2002; Wijnands, Miller, \& Wang 2002). The $0.5-10 \mathrm{keV}$ luminosity of the $\gamma$-ray source GRO J174428 during the survey was only $2-4 \times 10^{33} \mathrm{erg} \mathrm{s}^{-1}$ (assuming that the source is physically near the GC), compared to the earlier ROSAT detection of $2 \times 10^{37} \mathrm{erg} \mathrm{s}^{-1}$ in the $0.1-2.4 \mathrm{keV}$ band. The Chandra survey observation of SAX J1747.0-2853, with a $0.5-10 \mathrm{keV}$ luminosity of $3 \times 10^{35} \mathrm{erg} \mathrm{s}^{-1}$, was taken between two outbursts in 2000 and 2001. Another potentially interesting source is CXOGCS J174502.3-285450, which appeared orders of magnitude brighter in another Chandra observation taken about a year earlier than the survey. The "quiescent" properties of these transient sources can now be used to place 


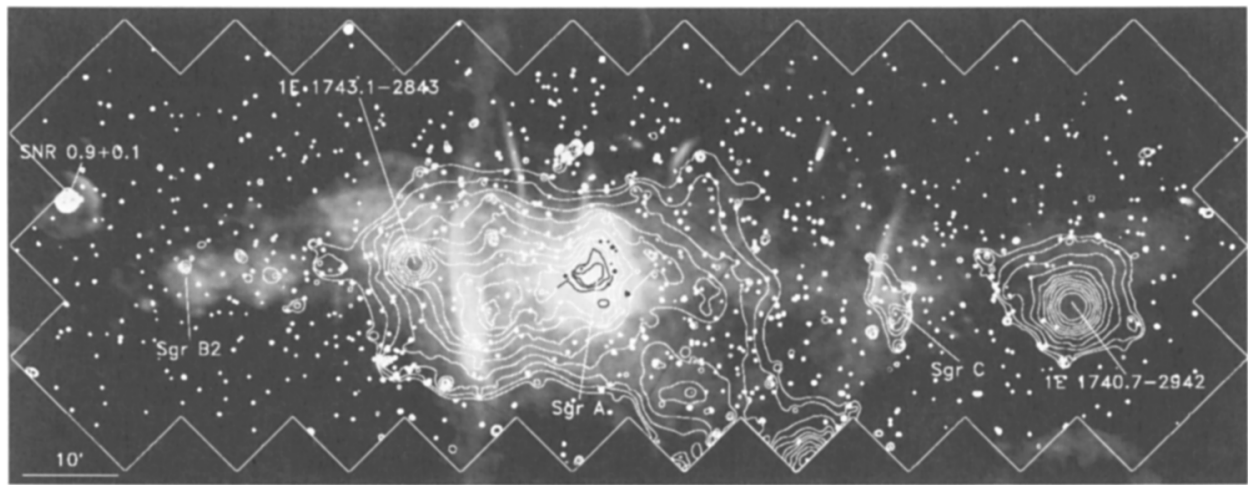

Figure 1. Chandra ACIS-I intensity contours overlaid on the radio $(90 \mathrm{~cm})$ image of the GC ridge. The X-ray intensity is calculated in the 1-8 keV range and is adaptively smoothed with a signal-to-noise ratio of $\sim 3$. The saw-shaped boundaries of the map, plotted in Galactic coordinates, results from a specific roll angle of the observations.

interesting constraints on the cooling process of neutron star surfaces and on the X-ray emission mechanism of neutron stars in poorly studied state of low accretion rates. The accurate Chandra positions of the sources will also make it much easier to identify counterparts at other wavelengths.

What are the origins of those newly detected and relatively faint X-ray sources? A considerable fraction (up to 30\%) of the sources are bright in 1-3 $\mathrm{keV}$ band and thus must be foreground objects (e.g., cataclysmic variables and normal stars). Many of the soft X-ray bright foreground stars are associated with two stellar clusters embedded within known optical emission nebulae Sh220 and Sh2-17 (Dutra \& Bica 2000 and references therein; Wang et al. 2002). Sources that are located physically near the GC or beyond are subject to Xray absorption of equivalent total gas column density greater than a few times $10^{22} \mathrm{H}$ atoms $\mathrm{cm}^{-2}$ and must show little or no soft X-ray emission/flux. A preliminary estimate based on millimeter and far-infrared emissions in the GC field suggests that less than a few percents of all these sources were extragalactic in origin. Therefore, the bulk of the sources are likely located in the GC region.

Pfahl et al. (2002) proposed that many of the sources may be wind-accreting neutron stars. An infrared observing campaign is now under way to search for the predicted stellar counterparts of the hard X-ray sources. The confirmation or rejection of a proposal like this is especially important for massive binary population synthesis studies.

\subsection{Sgr $\mathbf{A}^{*}$}

The single most important discovery made by Chandra in the GC region is probably the rapid X-ray flaring from Sgr A*, which hosts the central black hole of $\sim 3 \times 10^{6} \mathrm{M}_{\odot}$ (Baganoff et al. 2001). The flare detected in 2000 lasted for 
about 3 hours and exhibited variability on time scales of a few minutes. The Xray emission of the flare, characterized by a power law spectral model, has a peak $2-10 \mathrm{keV}$ luminosity of $\sim 1 \times 10^{35} \mathrm{erg} \mathrm{s}^{-1}$, or about 45 times the quiescent-state luminosity of Sgr $\mathrm{A}^{*}$. The detection of such a flare provides the most compelling evidence that matter falling toward the black hole is fueling energetic activity.

However, the nature of the quiescent-state X-ray emission, which is slightly extended on a scale of $\sim 1^{\prime \prime}$, remains unclear. The X-ray spectrum shows evidence for an emission line at $\sim 6.6 \mathrm{keV}$ and a characteristic thermal plasma temperature of $\sim 4 \mathrm{keV}$. The faintness of the emission, together with the lin-

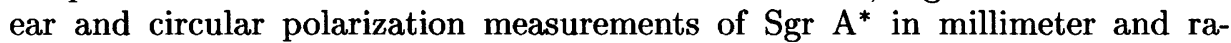
dio wavelengths, places tight constraints on the GC black hole accretion rate, $3-4$ orders of magnitude less than the expected Bondi accretion rate of $\sim$ $10^{-4}-10^{-5} \mathrm{M}_{\odot} \mathrm{yr}^{-1}$. Furthermore, a considerable fraction of the quiescentstate emission should be due to normal stars in the GC stellar cluster and possibly to the expected presence of numerous compact stellar remnants, e.g., millisecond pulsars around the black hole, as in globular clusters (e.g., Grindlay et al. 2001). Such stellar remnants, if present, may generate powerful relativistic winds that could significantly reduce the amount of gas falling into the black hole.

\subsection{Arches Cluster}

Chandra has also detected the strong X-ray emission from the Arches stellar cluster and its vicinity (Wang et al. 2002; Yusef-Zadeh et al. 2002). Located at only $12^{\prime}$ away from Sgr A*, the cluster contains more than 100 O-type and Wolf-Rayet stars within a region of $\lesssim 1$ pc across (Figer et al. 1999; Fig. 2). At an age of $1-2 \times 10^{6}$ years, the cluster is responsible for the ionization of the Arched filaments - the most prominent thermal features in the GC region. The Arches cluster is thus an excellent testbed for understanding physical processes involved in a compact young massive stellar cluster and in its interplay with the unique GC environment.

Fig. 2 shows three distinct X-ray sources/peaks apparently associated with the cluster. The spectra of these sources exhibit the prominent $\mathrm{K} \alpha$ emission lines of highly-ionized ions such as Ca XIX and Fe XXV (Yusef-Zadeh et al. 2002). Each source has an unabsorbed X-ray luminosity of $\sim 10^{35} \mathrm{ergs} \mathrm{s}^{-1}$ in the $0.2-$ $10 \mathrm{keV}$ band. There is also a very extended diffuse X-ray component with a luminosity of $\sim 2 \times 10^{34} \mathrm{ergs} \mathrm{s}^{-1}$. The spectrum of this component presents a strong 6.4-keV emission line with an equivalent width of $\sim 1 \mathrm{keV}$ (Yusef-Zadeh et al. 2002; see also Fig. 2).

The nature of the X-ray emission from the Arches cluster remains largely uncertain. A considerable fraction of the emission may come directly from individual massive stars. The total luminosity of the cluster $\left(L_{b o l} \sim 4 \times 10^{41} \mathrm{ergs} \mathrm{s}^{-1}\right.$; Figer et al. 1999) and the "canonical" relation between X-ray and total luminosity of $L_{x} / L_{b o l} \sim 10^{-7}$ (Chlebowski 1989) predicts an integrated X-ray luminosity $L_{x} \sim 4 \times 10^{34} \mathrm{ergs} \mathrm{s}^{-1}$, or about $10 \%$ of the observed X-ray luminosity of the Arches cluster. This fraction could be higher if the relation under-estimates the X-ray luminosity of stars with high metal abundances $(\sim 3 \times$ solar $)$ and in a dense stellar environment (e.g., Chlebowski 1989). 


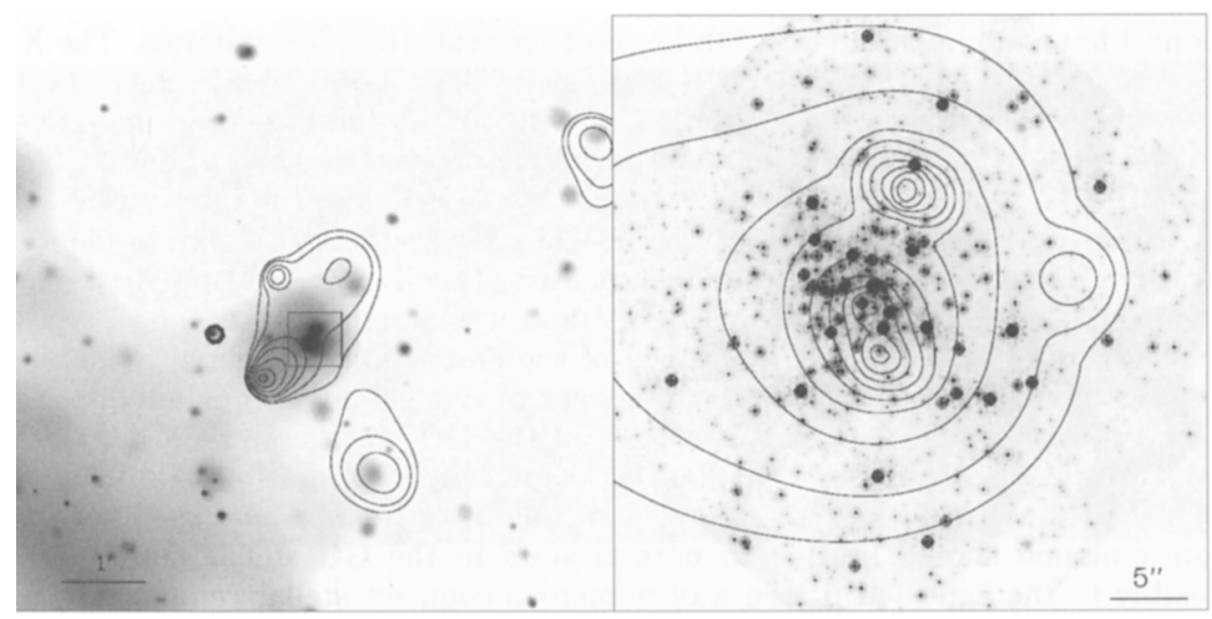

Figure 2. Left panel: The Arches cluster and its vicinity. The grey-scale image represents the Chandra ACIS-I image in the 1-8 keV band, while the overlaid contours illustrate the the $6.4-\mathrm{keV}$ line intensity distribution. Right panel: The close-up of the region outlined by the central box in the left panel. The contours are from the 1-8 keV band image and the grey-scale image from an HST NICMOS near-IR observation (Figer et al. 1999).

High X-ray luminosity sources may represent colliding winds in close binaries of extremely massive stars (Portegies Zwart et al. 2001). However, the $\mathrm{X}$-ray centroids of the sources in the Arches cluster do not match the positions of the brightest near-infrared stars (Fig. 2), which typically have the strongest stellar winds (Lang et al. 2001).

Alternatively, the X-ray sources may represent diffuse emission peaks of the so-called cluster wind (Raga et al. 2001; Yusef-Zadeh et al. 2002). Because of the high density of the massive stars, their stellar winds collide with each other and can be partly thermalized to an initial temperature of a few times $10^{7} \mathrm{~K}$. The expanding of this hot gas may be considered as a wind from the entire cluster. $\mathrm{X}$-ray emission from the cluster wind of the Arches cluster has been simulated by Raga et al. (2001). While it is not difficult to tune the stellar wind velocity and intensity to match the characteristic temperature and luminosity inferred from the X-ray data, the spatial distribution of the simulated X-ray morphology does not seem to match the observation. For example, the observed bright northern source, clearly resolved, is totally absent in all three simulations with assumed different line-of-sight distributions of cluster stars. The simulated X-ray emission also appears considerably less extended than the observed, linearly by a factor of $\sim 2$. Furthermore, the strong $6.4-\mathrm{keV}$ fluorescence line from cold Fe atoms in the spectrum of the diffuse X-ray emission is inconsistent with the hot thermal plasma model of the cluster wind.

The 6.4-keV line and much of the diffuse emission are most likely due to the X-ray fluorescence/scattering of the cluster X-ray emission by dense and cold gas in the vicinity. Indeed, the emission seems to coincides spatially with 
the southern tip of a dense molecular cloud observed in the region (Serabyn \& Güsten 1987). An ongoing in-depth study with recently obtained high resolution data on the cloud will allow for a detailed modeling of the fluorescence/scattering processes.

\subsection{X-ray Threads}

Chandra observations have revealed a number of very interesting linear X-ray features, which we call X-ray threads. They are oriented more-or-less vertically with respect to the Galactic plane, in a fashion similar to the radio bright NTFs. Radio polarization measurements show that the NTFs represent synchrotron radiation from relativistic particles trapped in magnetic field flux tubes. But the origins of both the magnetic field and the particles are still a mystery.

What might be the relationship between the radio NTFs and the X-ray threads? Interestingly, the three brightest X-ray threads (G0.13-0.11, G359.890.08 , \& G359.55+0.17) all seem to be related to either NTFs or nonthermal radio "wisps". The X-ray spectra of these threads can be characterized by a simple power law, consistent with being nonthermal in nature. Wang, Lu, \& Lang (2002) have proposed that G0.13-0.11 may represent the leading-edge of a pulsar wind nebula, The putative pulsar, identified as a point-like X-ray source embedded in the thread, is probably moving in a strong magnetic field environment. The main body of this pulsar wind nebula is likely traced by a bowshaped radio feature, which is apparently bordered by G0.13-0.11 and is possibly associated with the prominent NTFs of the Radio Arc. The high energy pulsar wind and the subsequent reverse-shock particle acceleration provide a natural explanation for the ultra-relativistic particles $\left(\sim 10^{14} \mathrm{eV}\right)$ required for producing synchrotron X-rays.

A similar interpretation may also apply to G359.55+0.17 ( $\mathrm{Lu}$, Wang, \& Lang 2002). This X-ray thread lies between an elongated nonthermal radio "wisp" and a possible point-like X-ray source (CXOGCS J174539.7-290413). In this case, the pulsar may be moving in a direction nearly parallel to magnetic field lines in the region. The ram-pressure confinement of the shocked pulsar wind material can then produce the linear structure formed by the X-ray source, the X-ray thread, and the radio wisp. The offset between the radio and X-ray features, for example, can be naturally explained by the synchrotron lifetime difference of the corresponding electrons/positrons.

Even more intriguing is the X-ray thread G359.54+0.18, which coincides exactly with the brightest section of one of the two adjacent and parallel NTFs (Wang 2002). While inverse self-Compton scattering is found unimportant, the $\mathrm{X}$-ray emission may also be synchrotron in nature. However, it is not clear what might be the origin of the required ultra-relativistic particles. A recent magnetic field reconnection between the two NTFs, as indicated by their close proximity, may be a possibility.

\subsection{Diffuse X-ray Emission}

The total count contribution from detected discrete sources from the GC region,

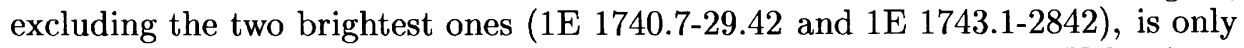
about $10 \%$ of the remaining X-ray flux, which has a total $2-10 \mathrm{keV}$ luminos- 

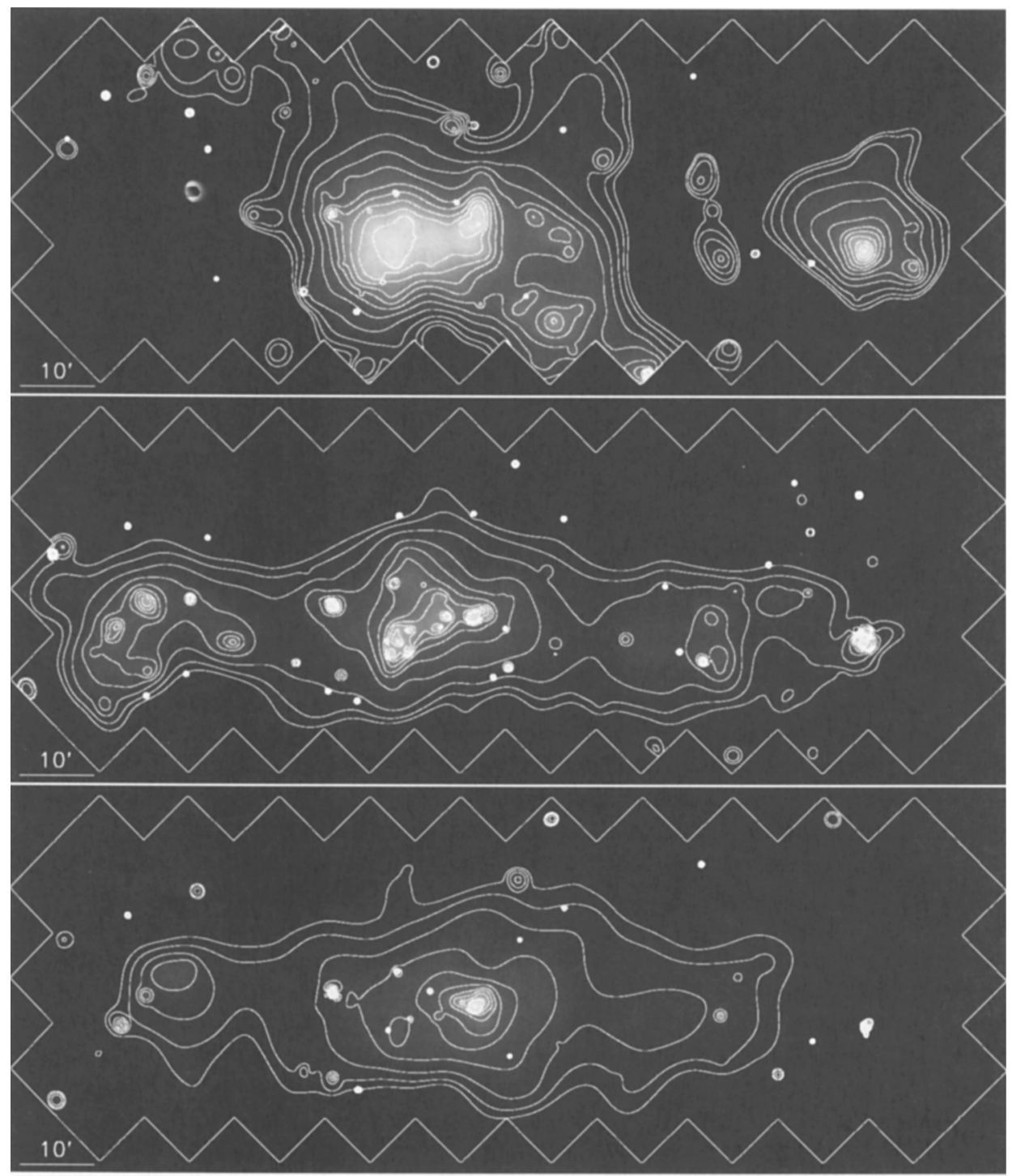

Figure 3. Narrow-band Chandra ACIS-I intensity maps in the 2.36$2.56 \mathrm{keV}, 6.2-6.55 \mathrm{keV}$, and 6.55-6.9 keV ranges (upper, middle, and lower panels), which are dominated by emission lines of $\mathrm{S} \mathrm{XV} \mathrm{K}_{\alpha}$, cold $\mathrm{Fe} \mathrm{K}_{\alpha}$, and $\mathrm{Fe} \mathrm{XXV} \mathrm{K}_{\alpha}$, respectively. The rest is the same as in Fig. 1.

ity of $\sim 10^{37} \mathrm{ergs} \mathrm{s}^{-1}$. What might be the origins of this apparently diffuse component? 
- The component cannot be explained by any known populations of X-ray sources with individual fluxes below our detection limit $\left(L_{x} \sim 10^{32-33}\right.$

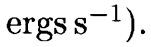

- The spectrum of the component shows strong $\mathrm{S} \mathrm{XV} \mathrm{K}_{\alpha}$ and Ar XVII $\mathrm{K}_{\alpha}$ lines, relative to the Fe XXV $\mathrm{K}_{\alpha}$ line (Wang 2002; e.g., Fig. 3). This indicates that the diffuse soft $(\lesssim 4 \mathrm{keV}) \mathrm{X}$-ray emission is dominated by thermal plasma at temperatures $\lesssim 6 \times 10^{7} \mathrm{~K}$. This plasma, however, cannot account for all the continuum emission at higher energies.

- The lack of a detailed correlation of the X-ray emission with either radio continuum (except for G359.54+0.18; see §2.4) or near-infrared features further suggests that inverse Compton scattering is not important.

- The required total X-ray luminosity for producing the 6.4-keV line emission (e.g., Fig. 3) via fluorescence is about two orders of magnitude greater than what is observed. Of course, some X-ray transients could be very luminous in the past. Alternatively, Sgr A* could have a luminosity of several times $10^{39} \mathrm{ergs} \mathrm{s}^{-1}$ about 300 years ago, or a factor of $\sim 10^{6}$ brighter than the average luminosity at present (Koyama et al. 1996). However, this scenario would predict an intensity correlation of the $6.4-\mathrm{keV}$ line emission with molecular tracers. This is not apparent at least in parts of the GC region (Wang 2002).

- The 6.4-keV line emission may arise from the filling of K-shell vacancies generated by collisions between cold iron atoms and low energy cosmic rays (Valinia et al. 2000). The bremsstrahlung of these cosmic rays may also be responsible for much of the diffuse hard X-ray continuum emission. A detailed modeling of this process will place interesting constraints on the largely-unknown population of low energy cosmic rays in the Galaxy.

The X-ray mapping of the GC region, together with observations of many other objects in the Galaxy, has allowed for detailed investigations into various high energy astrophysical phenomena and processes. But to gain galaxy-wind perspectives, we should resort to observations of nearby galaxies.

\section{Nearby Galaxies}

Chandra has made numerous observations of nearby galaxies, from dwarf irregulars to giant ellipticals. These observations allow for examining the interplay between various galactic components as well as for studying populations of Xray sources at the same distances to individual galaxies and with minimum line-of-sight confusion. I comment here on several such topics.

\subsection{X-ray Source Populations}

Even with the Chandra capability, we typically only detect X-ray sources with luminosities $\gtrsim 10^{37} \mathrm{erg} \mathrm{s}^{-1}$ in nearby galaxies outside the Local Group. Such $\mathrm{X}$-ray sources are mostly X-ray binaries with neutron stars or black holes as the primaries. The secondaries of the binaries are either high mass stars (HMXBs 
with lifetimes of $\sim 10^{7} \mathrm{yrs}$ ) or low mass stars (LMXBs with timescale of $\sim 10^{9}$ yrs). The relative populations of these two distinct types of X-ray binaries, especially for the luminous ones, naturally depend on galaxy types: starburst galaxies are prevailed by HMXBs whereas early-type galaxies are dominated by LMXBs. What do we learn from Chandra observations?

- The high resolution observations of Chandra have allowed for the first time to reliably construct the X-ray source luminosity function, $N-\log$ $S$, for individual galaxies. It is found that the $N-\log S$ slope flattens with increasing star formation rate of spiral galaxies (Kilgard et al. 2002; Swartz et al. 2002), which is consistent with the assumption that the more luminous HMXBs are shorter-lived. Therefore, the slope, or the high luminosity cutoff, of the luminosity function can be used as a measure of the massive star formation history of a galaxy (e.g., Wu 2001). However, the formation and evolution of LMXBs are believed to be quite different from those of HMXBs, which can seriously complicate the measurement.

- Large populations of LMXBs have been revealed in elliptical galaxies (e.g., Sarazin, Irwin, \& Bregman 2000; Kraft et al. 2001; Angelini et al. 2002). White, Sarazin, \& Kulkarni (2002) find that the ratio of the global LMXB luminosity to the galactic optical luminosity is not correlated with optically-derived stellar age indicator and, instead, is strongly correlated with the specific globular cluster frequencies in elliptical galaxies. In an HST-imaged field of NGC 1399, for example, Angelini et al. (2001) show that globular clusters account for $\sim 70 \%$ of X-ray sources with luminosities $\gtrsim 5 \times 10^{37}$ ergs s $^{-1}$. This suggests that most LMXBs were formed in globular clusters.

- $\log N-\log S$ relations for some elliptical galaxies show a break approximately at the Eddington limit $\left(\sim 2 \times 10^{38} \mathrm{ergs} \mathrm{s}^{-1}\right)$ on the isotropic luminosity for accretion onto a $1.4 M_{\odot}$ neutron star (Sarazin et al. 2000; Finoguenov \& Jones 2002). This break, if confirmed, may be used as a distance indicator for nearby galaxies.

\subsection{Ultra-Luminous $X$-ray Sources}

A considerable number of X-ray sources outside galactic nuclei appear to have luminosities substantially greater than the Eddington limit for a few solar mass black hole, assuming that the emission is isotropic. Some of these sources have luminosities as high as a few times $10^{40} \mathrm{ergs} \mathrm{s}^{-1}$. X-ray sources of this type, known for more than 20 years since the detections with Einstein, are often called ultra-luminous X-ray sources (ULXs) or super-Eddington sources. The most commonly-adopted interpretation for ULXs assumes that they are binary systems containing the so-called intermediate-mass black holes $\left(M \sim 10^{2}-10^{5} M_{\odot}\right)$. Alternatively, the apparent high X-ray flux may arise from highly beamed emission from accretion disks around stellar mass black holes or neutron stars the micro-quasar scenario (e.g., Markoff, Falcke, \& Fender 2001; King 2002). Furthermore, the binaries could also be truly super-Eddington, for example, obtainable from accretion disks with radiation-driven inhomogeneities (Begelman 2002). Much of the current debate is driven by new X-ray observations made 
with both Chandra and XMM-Newton. Let us see how such scenarios may confront the observations:

- Aperiodic variability by a factor greater than 2 and on various timescale scales has been detected for several ULXs (e.g., Mukai et al. 2002; Pence et al. 2001; Strickland et al. 2001). Such strong variability is expected for the relativistic beaming (jet) models.

- Two ULXs have been shown to vary periodically (Bauer et al. 2001; Liu et al. 2002). For example, a period of 7.5 hours is found for a ULX in the Circinus Galaxy. This ULX has a mean X-ray luminosity of $3.4 \times$ $10^{39} \mathrm{erg} \mathrm{s}^{-1}$ and an X-ray spectrum well fitted by a multicolor blackbody accretion disk model. These properties are consistent with an eclipsing $\gtrsim 50 M_{\odot}$ black hole binary (Bauer et al. 2001). The implied viewing angle, nearly edge-on, can probably be used to rule out the relativistic beaming model for such ULXs, because a jet should be oriented in the direction perpendicular to an accretion disk and hence should not be pointed to us.

- Most of ULXs have remained remarkably steady throughout their observed history, which goes back as long as 20 years ago when Einstein Observatory made the first detections (e.g., Swartz et al. 2000). This persistence of source fluxes poses difficulties for the jet models, especially ones that require large relativistic boosting of X-ray fluxes.

- Multiple observations show that some ULXs (e.g., Kubota et al. 2001; Liu, Bregman, \& Seitzer 2002) switched between high/soft and low/hard spectral states. These state changes are characteristic of Galactic accreting black hole binaries.

- An increasing number of ULXs have been identified in other wavelength bands (e.g., Wu et al. 2002; Liu et al. 2002). In particular, many of ULXs in elliptical galaxies are found within globular clusters. Interestingly, black holes with masses $\sim 4 \times 10^{3} M_{\odot}$ and $2 \times 10^{4} M_{\odot}$ have been discovered recently at the centers of the globular clusters M15 in our Galaxy (Gerssen et al. 2002) and G3 in M31 (Gebhardt, Rich, \& Ho 2002). Therefore, intermediate mass black holes can originate in globular clusters, many of which may have been tidally disrupted over the history of a galaxy.

- Many ULXs are located within shell-like optical nebulae (e.g., see the Pakull's contribution in this volume). The nebula around the variable ULX in dwarf galaxy Holmberg II, in particular, shows strong $4686 \AA$ recombination radiation from HeII atoms. The ionization of the atoms requires a soft X-ray luminosity that is consistent with the isotropic emission of the ULX.

Therefore, ULXs may represent a heterogeneous type of X-ray sources. It appears that accreting intermediate-mass black holes are a viable interpretation of most ULXs, although rapid varying objects and/or transients may be more naturally explained by the jet-induced beaming models or possibly by the superEddington scenarios. 


\subsection{Hot Gas Outflows from Nuclear Starburst Galaxies}

The extreme behavior of the violent interplay between massive stars and the ISM is the gas ejection from galactic disks. Naturally, most of the work on this topic has been concentrated on edge-on disk galaxies, which allow for detection of extraplanar gas unambiguously. Vertical X-ray plumes are detected along the minor axes of many nuclear starburst galaxies (e.g., NGC 253, Strickland et al. 2002; NGC 3079, Cecil et al. 2002; NGC 4945, Weaver 2001; M82, Lehnert, Heckman, \& Weaver 1999, Fig. 4). These diffuse X-ray plumes are typically characterized by soft thermal spectra of plasma with steep temperature gradients in the disk/halo transition zones close to the nuclear regions. Morphologically, the diffuse X-ray plumes are often double horn-shaped and are well correlated with extraplanar $\mathrm{H} \alpha$ features.
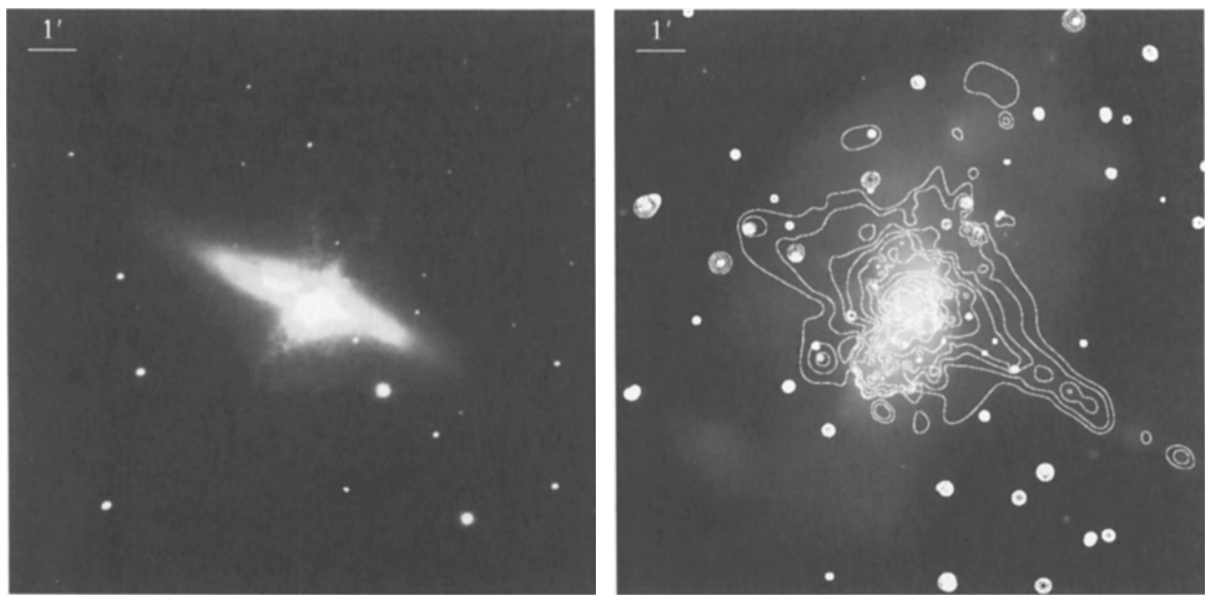

Figure 4. M82 in $\mathrm{H} \alpha$ (left panel; Hintzen et al. 1993) and in X-ray (right panel). The X-ray intensity image is in the $0.3-0.7 \mathrm{keV}$ band, whereas the contours are in $0.7-1.5 \mathrm{keV}$ band. The linear extension toward the southwest (the CCD readout direction), nearly parallel to the disk of the galaxy, is partly an artifact.

In comparison, outflows driven by AGNs are not necessarily along the minor axes of galaxies and are primarily in form of jets or well-collimated/centrallyfilled lobes (e.g., NGC 1068; Young, Wilson \& Shopbell 2001). The X-ray emission from AGN-induced outflows is due to hot gas heated by the terminal shock of the outflows (e.g., Terashima \& Wilson 2001).

Deep X-ray observations of nuclear starburst galaxies have further revealed large-scale, low surface brightness diffuse X-ray halos, which seem to be confined within faint limb-brightened $\mathrm{H} \alpha$-emitting boundaries (e.g., NGC 253, Strickland et al. 2002; NGC 1569, Martin et al. 2002; M82, Lehnert et al. 1999, Fig. 4).

Extraplanar diffuse X-ray emission has also been observed around relatively normal galaxies, in which star formation is not concentrated in nuclear regions. The best case for the presence of a diffuse hot gaseous halo is the edge-on Scd galaxy NGC 4631 (Wang et al. 2001). The diffuse X-ray emission is very soft and extends up to about $10 \mathrm{kpc}$ away from the galactic plane. The overall $\mathrm{X}$ - 
ray emission morphologically resembles the well-known radio halo of the galaxy, indicating a possible link between outflows of hot gas and cosmic ray/magnetic field from the galactic disk. Substantial amounts of extraplanar diffuse X-ray emission have also been detected around another nearby Sc galaxy NGC 3556 (Wang 2002). Its radio and X-ray halos are also morphologically similar.

The most logical explanation for the characteristics of the hot gas outflows and halos is a confined galactic wind model (e.g., Strickland et al. 2002). A free-streaming wind is not efficient in radiating X-rays, because it is cooled and rarefied rapidly during the adiabatic expansion phase within or just outside the galactic disks or nuclear regions. Thus, the X-ray emission that spatially correlates with $\mathrm{H} \alpha$ features does not directly arise from fast outflowing galactic winds. The emission may instead come from shocked and/or dynamically mixed gas around pre-existing clouds or at the interfaces between the winds and the galactic gaseous disks. If sufficient amounts of cool gas are present in the halos, as indicated by the outer $\mathrm{H} \alpha$-emitting boundaries observed, the galactic wind may be thermalized again and may be mass-loaded by thermal conduction at the boundaries. The thermalized plasma can thus be responsible for the large-scale, low surface brightness X-ray emission seen around M82, for example (Fig. 4). Of course, if the plasma is hot enough and does not cool sufficiently fast, it may eventually escape from galaxies due to the buoyancy and/or to the ram-pressure of the IGM.

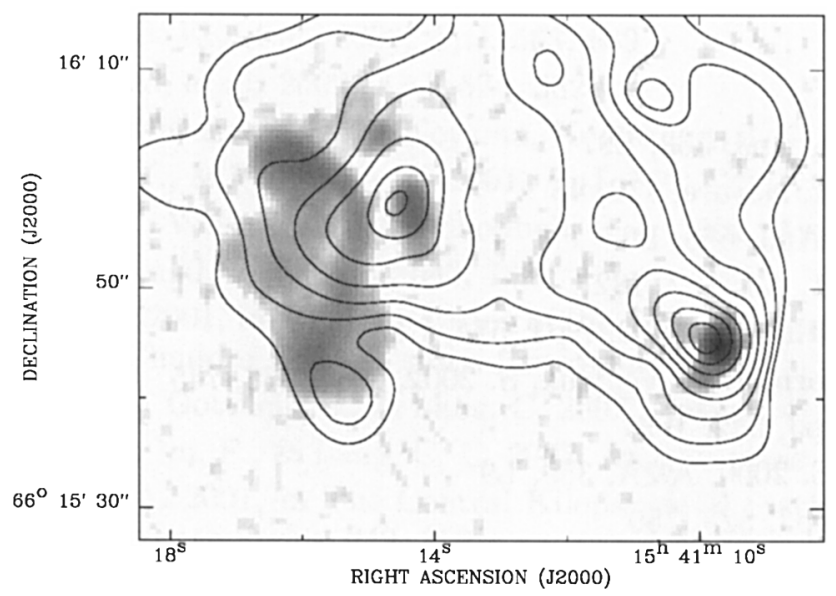

Figure 5. The central region of the cluster Abell $2125(z=0.25)$ in radio (gray-scale) and X-ray (contours). The X-ray intensity contours are at $14,15,18,20,24,28,33$, and $39 \times 10^{-3}$ cts s$^{-1} \operatorname{arcmin}^{-2}$ in the $0.5-4 \mathrm{keV}$ band. 


\subsection{Interplay of Galaxies with Their Environments}

The large amounts of gas in galactic halos, as inferred from the confinement of galactic winds, may represent the accretion of the IGM onto the galaxies in the group environment. The accretion is predicted by numerical simulations of the structure formation (e.g., Murali et al 2002) and is supported by lines of observational evidences (Burstein \& Blumenthal 2002). The accretion, likely consists of multiple gaseous phases, may also occur around our Galaxy, as indicated by the presence of high velocity HI clouds of low metallicity and by recent detections of OVI, OVII, and OVIII absorption lines with FUSE and Chandra (e.g., Nicastro et al. 2002). The interaction between the galactic outflows and accretion inflows can then be used to probe their physical and chemical properties.

The effect of the intergalactic gas ram-pressure can be seen most vividly in the rich cluster environment. Fig. 5 demonstrates the use of X-ray emission as a tracer for the effect (Wang et al. 2002, in preparation). The hot intracluster medium density clearly affects the morphology of extended radio lobes. Furthermore, an apparent X-ray-emitting wake, trailing the lower right galaxy in the figure, is likely due to the ram-pressure stripping of galactic cool gas and possible subsequent mixing with the hot ambient medium. An ongoing multiwavelength investigation will help to quantify the interaction between these galaxies and the cluster environment.

I thank the symposium organizers for inviting me to give this talk and my research collaborators for their contributions to the various projects mentioned above.

\section{References}

Angelini, L., et al. 2002, ApJ, 568, L97

Baganoff, F., et al. 2001, Nature, 413, 45

Begelman, M. C. 2002, ApJ, 568, L97

Bauer, F. E., et al. 2001, AJ, 122, 182

Burstein, D., \& Blumenthal, G. 2002, ApJ, 574, L17

Cecil, G., Bland-Hawthorn, J., \& Veilleux, S. 2002, ApJ, 576, 745

Chlebowski, T. 1989, ApJ, 342, 1091

Dutra, C. M., \& Bica, E. 2000, A\&A, 359, L9

Figer, D., et al. 1999, ApJ, 525, 750

Finoguenov, A., \& Jones, C. 2002, ApJ, 574, 754

Gebhardt, K., Rich, R. M., \& Ho, L. 2002, ApJ, 578, L41

Gerssen, J., et al. 2002, AJ, in press

Grindlay, J., Heinke, C., Edmonds, P., \& Murray, S. 2001, Science, 292, 2290

Helfand, D. J. 1984, ASPP, 96, 913

Hintzen, P., et al. 1993, In The Evolution of Galaxies and Their Environment, 38

Kilgard, R. E., et al. 2002, ApJ, 573, 138

King, A. R. 2002, MNRAS, 335, L13 
Koyama, K., et al. 1996, PASJ, 48, 249

Kraft, R. P., et al. 2001, ApJ, 560, 675

Kubota, A., et al. 2001, ApJ, 547, L119

Lang, C., et al. 2001, ApJ, 551, 143

Lehnert, M. D., Heckman, T. M., \& Weaver, K. A. 1999, ApJ, 523, 575

Liu, J. F., Bregman, J. N., \& Seitzer, P. 2002, ApJ, 580, L31

Liu, J. F., Bregman, J. N., Irwin, J. A., \& Seitzer, P. 2002, ApJ, in press

Lu, F., Wang, Q. D., \& Lang, C. C. 2002, ApJ, submitted

Markoff, S., Falcke, H., \& Fender, R. 2001, A\&A, 372, L25

Martin, C. L., Kobulnicky, H. A., \& Heckman, T. M. 2002, 574, 663

Morris, M. 1994, in The Nuclei of Normal Galaxies, p185

Mukai, K., et al. 2002, ApJ, in press (astro-ph/0209166)

Murali, C., et al. 2002, ApJ, 571, 1

Nicastro, F., et al. 2002, Nature, submitted (astro-ph/0208012)

Pence, W. D., et al. 2001, ApJ, 561, 189

Pfahl, E., Rappaport, S., \& Podsiadlowski, P. 2002, ApJ, 571, 37

Portegies Zwart, S. F., et al. 2002, ApJ, 565, 265

Raga, A. C., et al. 2001, ApJ, 559, 33

Sarazin, C. L., Irwin, J. A., \& Bregman, J. N. 2000, ApJ, 544, L101

Serabyn \& Güsten 1987, A\&A, 184, 133

Strickland, D. K., et al. 2001, ApJ, 560, 707

Strickland, D. K., et al. 2002, ApJ, 568, 689

Swartz, D. A., et al. 2002, ApJ, 574, 382

Takagi, S., Murakami, H., \& Koyama, K. 2002, ApJ, 573, 275

Terashima, Y., \& Wilson, A. S. 2001, ApJ, 560, 139

Young, A. J., Wilson, A. S., \& Shopbell, P. L. 2001, ApJ, 556, 6

Valinia, A., et al. 2000, ApJ, 543, 733

Wang, Q. D. 2001, in The New Vision of the X-ray Universe in the XMM-Newton and Chandra Era", in press (astro-ph/0202317)

Wang, Q. D., Gotthelf, E., \& Lang, C. 2002, Nature, 415, 148

Wang Q. D., Lu, F., \& Lang, C. C. 2002, ApJ, in press

Weaver, K. A. 2001, in The Central Kiloparsec of Starbursts and AGNs, APS Conference Series, 249, 389

White III, R. E., Sarazin, C. L., \& Kulkarni, S. R. 2002, ApJ, 571, L23

Wijnands, R., \& Wang, Q. D., 2002, ApJ, 568, 93

Wijnands, R., Miller, J. M., \& Wang, Q. D. 2002, ApJ, 579, 422

Young, Y., Wilson, A. S., \& Shopbell, P. L. 2001, ApJ, 556, 6

Yusef-Zadeh, F., et al. 2002, ApJ, 570, 665

White III, R. E., Sarazin, C. L., \& Kulkarni, S. R. 2002, ApJ, 571, L23

$\mathrm{Wu}, \mathrm{H}$., et al. 2002, ApJ, 576, 738

Wu, K. 2001, PASA, 18, 443 